\title{
Quantification and analysis of saccadic and smooth pursuit eye movements and fixations to detect oculomotor deficits
}

\author{
Christopher A. DiCesare ${ }^{1}$ - Adam W. Kiefer ${ }^{1,2,3}$ - Patrick Nalepka ${ }^{2}$. \\ Gregory D. Myer ${ }^{1,3,4,5,6}$
}

Published online: 24 December 2015

(C) Psychonomic Society, Inc. 2015

\begin{abstract}
Assessment of deficits in oculomotor function may be useful to detect visuomotor impairments due to a closed head injury. Systematic analysis schemes are needed to reliably quantify oculomotor deficits associated with oculomotor impairment via brain trauma. We propose a systematic, automated analysis scheme using various eye-tracking tasks to assess oculomotor function in a cohort of adolescents with acute concussion symptoms and aged-matched healthy controls. From these data we have evidence that these methods reliably detect oculomotor deficits in the concussed group, including reduced spatial accuracy and diminished tracking performance during visually guided prosaccade and self-paced saccade tasks. The accuracy and tracking deficits are consistent with prior studies on oculomotor function, while introducing novel discriminatory measures relative to fixation assessments - methodologically, a less complicated measure of performance - and thus represent a reliable and simple scheme of detection and analysis of oculomotor deficits associated with brain injury.
\end{abstract}

Christopher A. DiCesare

christopher.dicesare@cchmc.org

1 Division of Sports Medicine, Cincinnati Children's Hospital Medical Center, 3333 Burnet Ave., MLC 10001, Cincinnati, OH 45229, USA

2 Center for Cognition, Action, and Perception, Department of Psychology, University of Cincinnati, Cincinnati, OH, USA

3 Department of Pediatrics, College of Medicine, University of Cincinnati, Cincinnati, OH, USA

4 Department of Orthopaedic Surgery, College of Medicine, University of Cincinnati, Cincinnati, OH, USA

5 Sports Health and Performance Institute, The Ohio State University, Columbus, OH, USA

6 The Micheli Center for Sports Injury Prevention, Boston, MA, USA
Keywords Concussion · Oculomotor · Eye tracking · Saccade $\cdot$ Fixation $\cdot$ Smooth pursuit

\section{Introduction}

Assessment of oculomotor function with relatively simple and cost-effective approaches can give powerful insight into neurological deficits related to visuomotor processing and performance (Heitger, Anderson, \& Jones, 2002; Heitger et al., 2004). It is therefore becoming more common to integrate oculomotor assessments into evaluations of neurophysiological and neurodegenerative (Crawford et al., 2005) disorders and injury. Oculomotor assessments are especially useful for detecting deficits that occur as a consequence of brain injury; it has been established that closed head injury can result in significant impairments in oculomotor and visuomotor function (Cifu et al., 2015; Heitger et al., 2004; Suh, et al., 2006a, b). Specifically, these common oculomotor impairments include reductions in spatial accuracy of saccadic eye movements, increased lag during smooth pursuit tracking, reduced peak saccade velocities and accelerations (Cifu et al., 2015), and delays and errors in planning, decision-making and other executive functions (Heitger et al., 2002, 2004).

While impairments associated with brain injury are often acute, many individuals exhibit subacute and chronic impairments as well (Carroll et al., 2004; Heitger et al., 2006). Further, there is heightened potential for incurring additional and compounding injury, as well as longer recovery times, after suffering previous concussive events (Guskiewicz et al., 2003). It appears that head trauma reduces an individual's capacity for selectively discriminating between objects of interest (Heitger et al., 2002; Williams et al., 1997), and this may affect their ability to prepare for, and avoid, blows to the head that are otherwise preventable. Because of the demonstrable 
and significant cognitive impairments that arise from closed head injury, there is rationale for developing both effective preventative measures and accurate diagnostic tools for detecting loss of function. Quick and accurate oculomotor assessments are needed to effectively detect deficits and, by proxy, neurological impairment. Ultimately, these tools could distinguish individuals suspected to have a closed head injury, as well as characterize the trajectory of recovery for individuals who experienced head trauma.

Effective oculomotor assessments that incorporate both saccade and smooth pursuit tasks may be integral in quickly and accurately detecting impairments. It is important, therefore, to identify systematic methods of recording and analyzing gaze data from these tasks that can quantify the presence and/or extent of potential impairments. In this paper, we present simple analysis schemes for the automated optimization, reduction, and assessment of data recorded from eye-tracking tasks designed to distinguish impaired individuals through the detection of oculomotor deficits. Additionally, we show that these methods can detect specific deficits during both saccade and smooth pursuit eye-tracking tasks in individuals who recently suffered a concussive event relative to their healthy peers. Finally, we end with a discussion of the implications of these analyses as it pertains to closed head injury in sport and other performance-driven environments.

\section{Materials and methods}

\section{Participants}

Seventeen individuals (12 males, five females; mean age $16.8 \pm 1.2$ years) who had recently experienced and had been diagnosed with a concussion (15 participants; mean days since concussion $7.67 \pm 4.69$; exact dates from two participants were not obtained) and 17 age-matched controls (ten males, seven females; mean age $16.8 \pm 0.7$ years) were included in this study. The recently concussed group were reported to have a greater severity of symptoms compared to the control group, based on the PostConcussion Symptoms Inventory (PCSI), $F(1,30)=$ 16.84, $p<0.001, \eta^{2}=0.36$ (symptoms from two participants in the patient group were not obtained). Our sample size was based on the results of a similar study on patients with closed head injury (Heitger et al., 2004). A power analysis based on the reported eye-tracking measure from the random smooth pursuit movement was conducted to estimate the sample size to achieve $80 \%$ power with an alpha of .05 . The analysis revealed 15 participants for each group was adequate to provide $80 \%$ power and sensitive to a proposed difference of $15 \mathrm{~ms}$ in lag time.

\section{Eye-tracking setup}

Gaze data were collected at $60 \mathrm{~Hz}$ using a Tobii X2-60 Eye Tracker (Tobii, Stockholm, Sweden). The tracker was mounted to the bottom center of a 61-cm computer monitor (Fig. 1) that was situated on top of a desk. Participants were instructed to sit upright and place their arms and hands on their thighs. Participants were seated such that the center of the monitor was at eye level and their eyes were $65 \mathrm{~cm}$ away from the monitor. Participants were initially instructed to fixate at the center of the monitor, and their eye gaze was tracked and the height of the monitor was adjusted as needed to ensure their gaze was centered. Participants were then calibrated at this position using a five-point calibration during which participants were instructed to fixate on a target that moved randomly between five pre-determined points with the target remaining static at each location for $2 \mathrm{~s}$. The five points were located at the center of the monitor and each of the four corners, at which the margins were $10 \%$ of the width and height of the monitor. Participants were instructed to restrict their movement as much as possible throughout the assessment. If at any point throughout the assessment the subject moved beyond any normal postural sway necessary to maintain an upright seated position, like a shifting of the subject's weight or an involuntary action like coughing or sneezing, the subject was re-aligned with the monitor and re-calibrated.

\section{Eye-tracking tasks}

For all tasks, participants tracked a dark gray circular target (RGB color model $=\left[\begin{array}{lll}0.5 & 0.5 & 0.5\end{array}\right]$ ) with a black crosshair in the center of the target against a light gray background (RGB color model $=\left[\begin{array}{lll}0.94 & 0.94 & 0.94\end{array}\right]$ ) using only their eyes. The target was $4 \mathrm{~cm}$ in diameter, and the crosshair was $1 \mathrm{~cm}$ in diameter. This target size was selected to prevent exacerbation of symptoms for the patients with concussion. In particular, it is well known that physical symptoms such as headache, nausea, and dizziness persist in the days and weeks after acute concussion. Because of this, we elected to display a larger target size with a smaller crosshair in order to promote finegrained oculomotor behavior while still presenting a stimulus that was tolerable for the patients (Grubenhoff, Kirkwood, Deakyne, \& Wathen, 2011; Lovell et al., 2003). Participants completed a battery of tests that included two 30-s tests of a prosaccade task, two 30-s tests of a self-paced saccade task (Fig. 2B), two tests each of a smooth pursuit task at three different angular velocities (Fig. 2C), and two tests of a smooth pursuit task (Fig. 2C) that randomly changed angular velocities (mean angular velocity $247.21 \pm 121.73^{\circ} * \mathrm{~s}^{-1}$ as presented to concussed patients; $252.03 \pm 135.32^{\circ} * \mathrm{~s}^{-1}$ as presented to healthy controls) for a total of 18 tests. A test was defined as a given iteration of a particular task (e.g., the prosaccade task), a task was defined as a series of trials, and a 


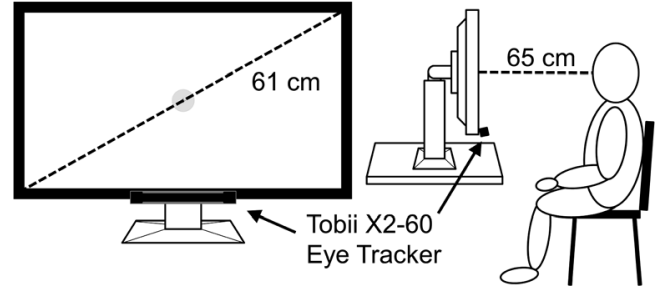

Fig. 1 Setup of the eye-tracking assessment including positions of the monitor, Tobii X2-60 Eye Tracker, and participant

trial was defined as a discrete action from which dependent variables were measured (e.g., a single step of the target during the prosaccade task).

For the prosaccade task, participants were told that the target would randomly step along a horizontal line and were

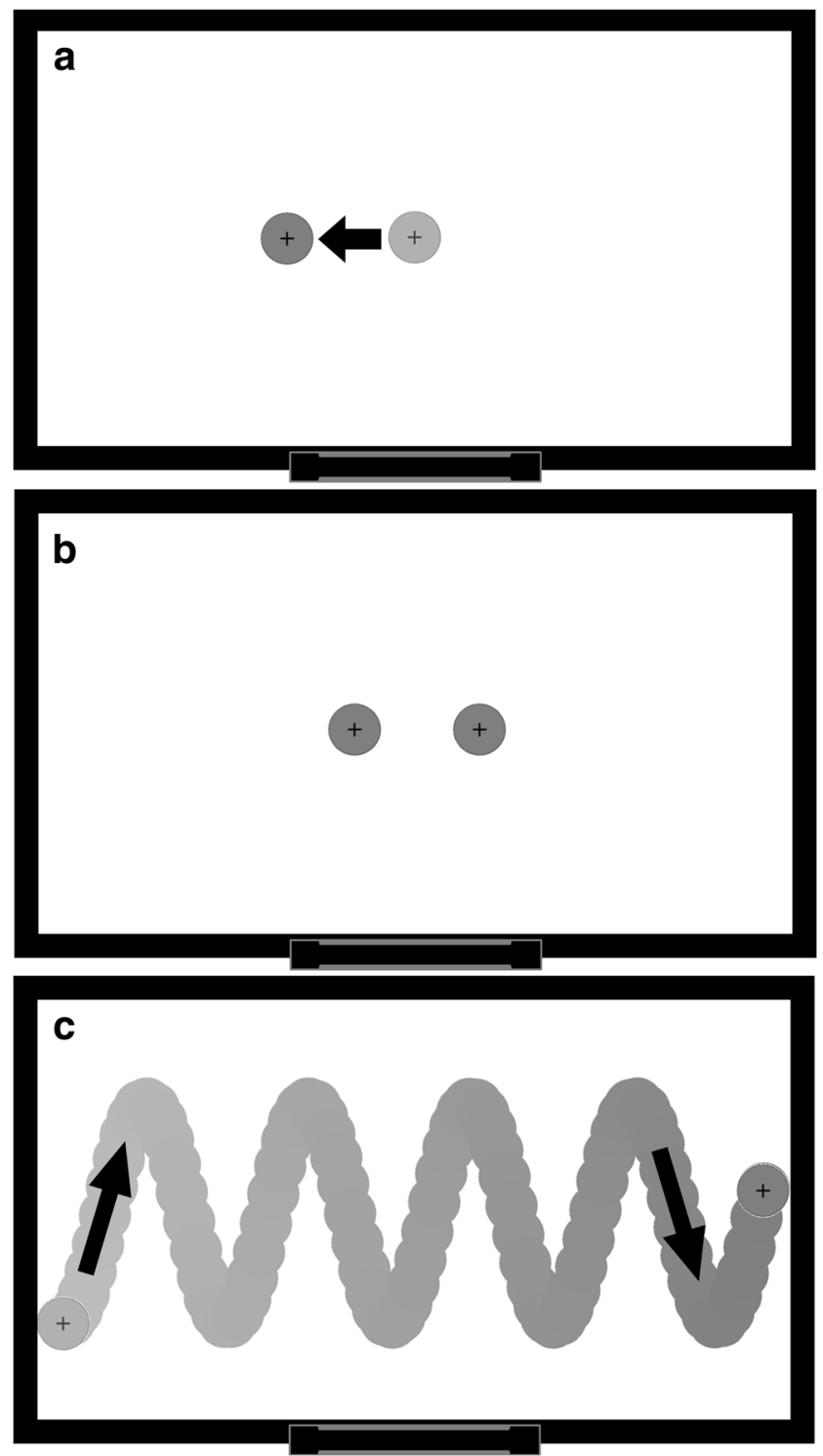

Fig. 2 Representative figure showing target positions for each of the eyetracking tasks. (A) An example target step at the onset of the prosaccade task; (B) the positions of the targets during the self-paced saccade task; and $(\mathbf{C})$ the movement of the target during the pursuit tasks instructed to keep their eyes focused in the center of the crosshairs in the target and to follow the target as it stepped. The target was initially displayed at the center of the monitor and, upon commencement of the task, stepped horizontally with a randomly determined magnitude between $4^{\circ}$ and $10^{\circ}$. The target would remain static after each step for a randomly determined period of time between 1 and $2 \mathrm{~s}$ and then step again.

For the self-paced task, the participants were told that two targets would be displayed on the screen but would not move. They were then instructed to look back and forth between the crosshairs in the middle of the left target and the crosshairs in the middle of the right target as quickly and accurately as possible. The two targets were displayed vertically at $50 \%$ of the height of the monitor and horizontally equidistant from the center of the monitor, $8^{\circ}$ apart.

For the smooth and random pursuit tasks, the participants were told that the target would be moving in a sinusoidal wave left-to-right across the screen. They were then instructed to keep their eyes focused on the crosshairs in the center of the target as closely as possible. The three target angular velocities indicated the length of time the target took to traverse a period of the of the sinusoidal path; for the "fast," "medium," and "slow" angular velocities, corresponding with angular velocities of $90^{\circ} * \mathrm{~s}^{-1}$, $180^{\circ} * \mathrm{~s}^{-1}$, and $360^{\circ} * \mathrm{~s}^{-1}$, respectively, the target took 1 , 2 , and $4 \mathrm{~s}$ to traverse a period, respectively. The tests were block randomized, i.e., participants would complete all tests of a randomly selected task before moving on to a different task. Before each task, the participant was instructed to fixate on the target for $2 \mathrm{~s}$, and the error of this fixation was calculated and displayed immediately to the tester. This was included as an added precaution for the tester to ensure quality data collection; typically, this error would increase if the participant's head moved between tests, the participant slouched in the chair, etc. In general, the tester only kept tests with an error of less than $1 \mathrm{~cm}$ and re-calibrated or had the participant redo the test if necessary.

\section{Gaze data analysis}

All data analyses were performed using custom Matlab scripts (Matlab, Natick, MA, USA). For all tasks, the gaze data from both eyes were initially converted into one data set by averaging the two-dimensional position of the gaze point of each eye relative to the computer monitor on which the tasks were displayed. This singular data set was then interpolated to remove small gaps. Linear interpolation was used to fill in missing data for gaps $\leq 67 \mathrm{~ms}$ (four samples). Afterward, the gaze data were smoothed using a nonweighted, symmetrical moving average filter with an averaging window of length $83 \mathrm{~ms}$ (five samples) (Olsen, 2012), in which samples before and after the current sample were 
Fig. 3 Gaze data (red) superimposed over the stimulus path (black) during a prosaccade task (top) and a smooth pursuit task (bottom). The prosaccade task is plotted as a function of horizontal monitor position and time, showing each step of the stimulus and subsequent gaze response. The smooth pursuit task is plotted as a function of horizontal and vertical monitor position. The top plot for each shows raw, unfiltered gaze data and the bottom shows interpolated, filtered gaze data
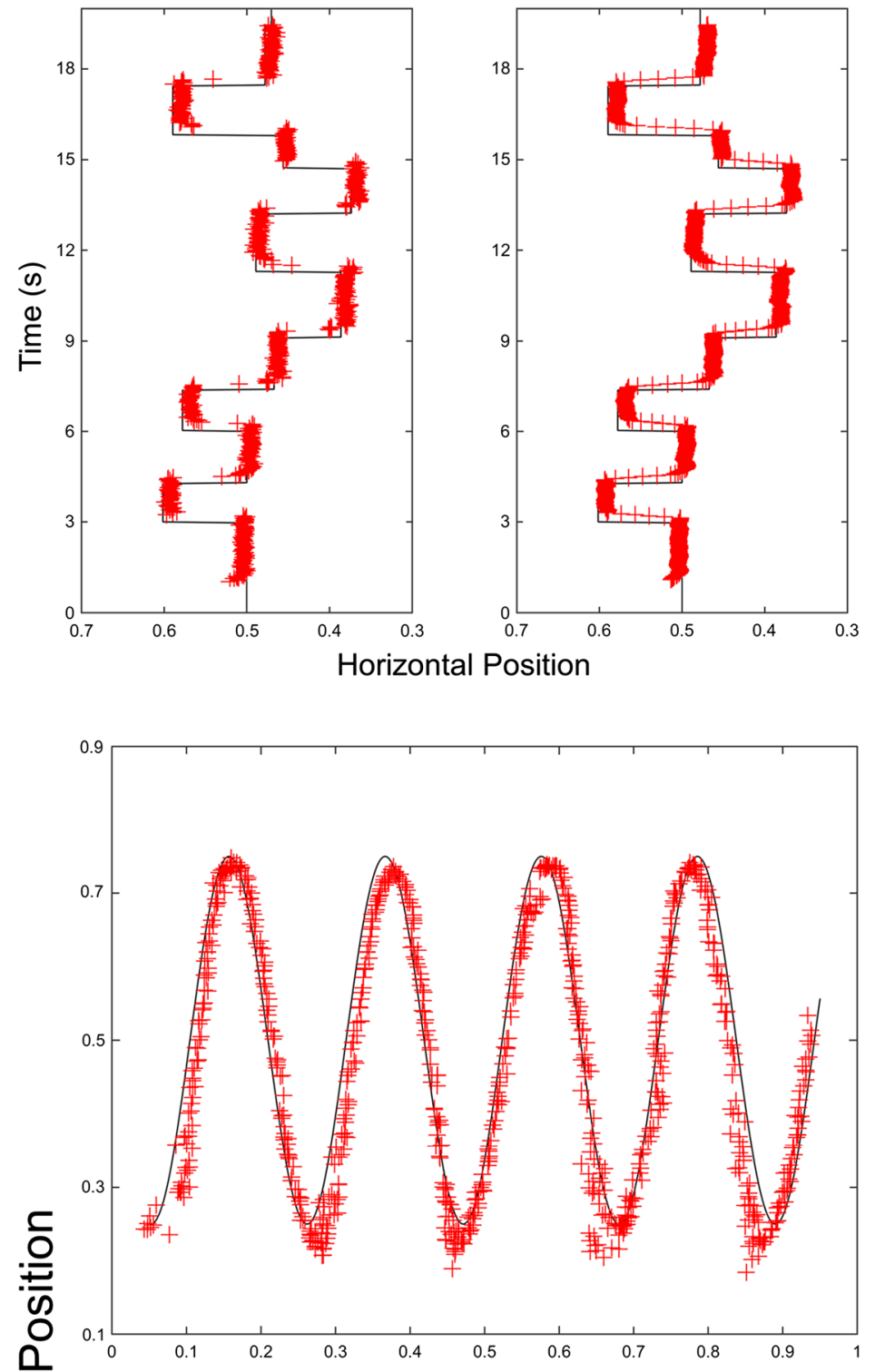

离

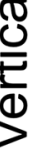

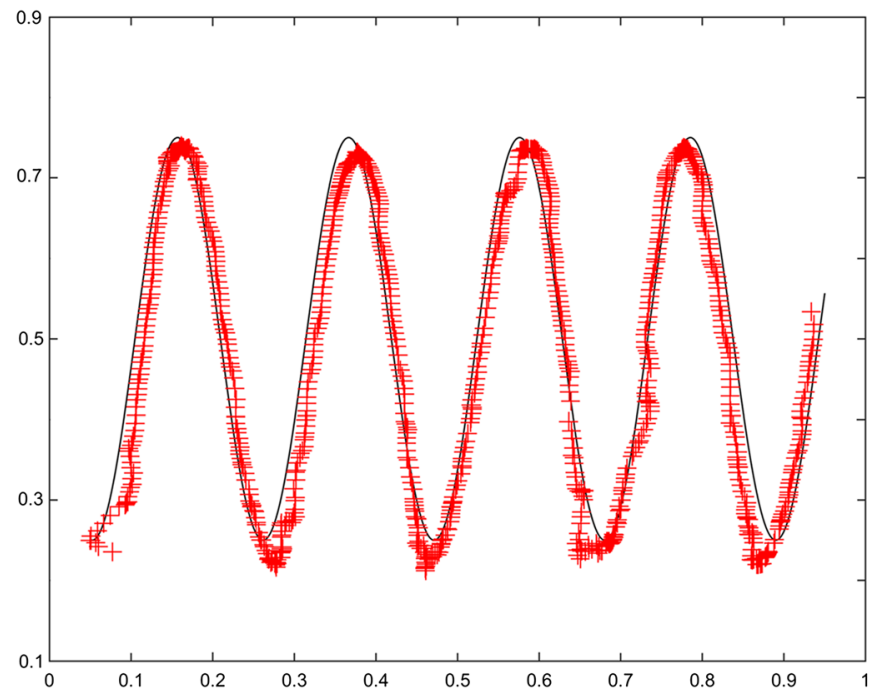

Horizontal Position 
used to produce an average at that position. The filter was adjusted in the case where fewer samples were present in the designated window, such as following or immediately preceding a gap in the data. For example, when the filter was applied following a gap in the data, the first frame was not averaged at all, the second frame was averaged using three frames - the first, second, and third frames following the gap - and the third frame was averaged using five frames the first, second, third, fourth, and fifth frames following the gap (Fig. 3). After interpolation and smoothing, the $\mathrm{x}$ - and $y$-coordinate positions of both the target and gaze data were converted to absolute measures of displacement (in $\mathrm{cm}$ ) relative to the center of the monitor screen.

\section{Saccade analysis}

For saccade tasks, velocity of gaze data were determined by first calculating the angular displacement of gaze position over subsequent samples for $N-1$ total calculations, where $N$ was the total number of gaze data samples recorded. This measure was dependent on both the displacement from the center of the monitor screen on both axes and the distance of the eyes from the screen (Fig. 4). This angular displacement was then converted to units per second.

Saccades were identified using a gaze velocity threshold of $30^{\circ} * \mathrm{~s}^{-1}$ (Fig. 5). The beginning of the saccade was designated at the point when the gaze velocity surpassed this threshold, and the ending of the saccade (and correspondingly, the beginning of fixation) was designated at the point when the velocity fell below this threshold. The latency of prosaccades was determined by comparing the beginning of the detected saccade to the corresponding change in position of the target. The dependent variables recorded for saccade analysis included the average latency over all saccade trials in each 30 -s prosaccade task, the total number of self-paced saccades in each 30-s self-paced saccade task, and the average saccade velocity over all saccade trials in each 30 -s prosaccade and self-paced saccade task.

\section{Fixation analysis}

Fixation error was calculated from the average, absolute displacement in centimeters between the gaze point and the center of the target over the duration of the fixation (from the ending of one saccade to the beginning of the next). The initial fixation error was designated as the error of the first gaze sample after the conclusion of a saccade. The dependent variables recorded for fixation analysis included the average fixation error and the average initial fixation error over all fixations in each 30 -s prosaccade and self-paced saccade task.

\section{Smooth pursuit analysis}

For smooth pursuit tasks, target and gaze position data were converted to a phase angle measure relative to the period of the sinusoidal wave over which the target traveled. The angular velocity of each was then determined by calculating the phase angular displacement over subsequent samples for $N-1$ total calculations, where $N$ was the total number of data samples recorded. This angular displacement was then converted to units per second. The phase lag was determined as the absolute difference between the stimulus phase angle and the gaze phase angle (Fig. 6). The dependent variables recorded for smooth pursuit analysis included the average phase lag and the average gaze angular velocity in each task of each condition.

\section{Statistical analysis}

For all dependent variables, outliers - designated as falling outside the range of the mean value $\pm 3 \mathrm{SD}$ - were removed before the final analysis. Standard t-tests assuming equal variance were used to assess group differences in measures of the number of self-paced saccades, prosaccade latency, saccade velocity, fixation error, and initial fixation error for saccade tasks, and phase lag and gaze angular velocity for pursuit tasks. An alpha level of .05 was used to indicate statistical significance.

\section{Results}

Table 1 displays the group differences between concussed participants and healthy controls in the dependent variables determined for the saccade tasks, as well as the $t$-values, $p$ values, $95 \%$ confidence intervals, and effect sizes $(d)$. There were significant differences in fixation and initial fixation

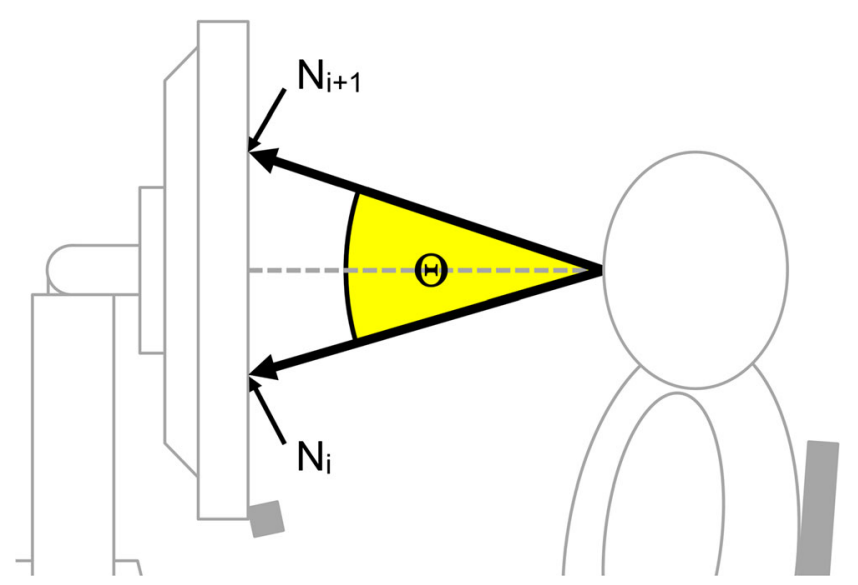

Fig. 4 Calculation of angular displacement over subsequent gaze data samples. The figure represents angular displacement for the y-axis only; however, angular displacement was ultimately calculated for both axes 
Fig. 5 Time-series curve showing gaze velocity for a selfpaced saccade task. The dotted red line indicates the threshold $\left(30^{\circ} * \mathrm{~s}^{-1}\right)$ used to detect saccades

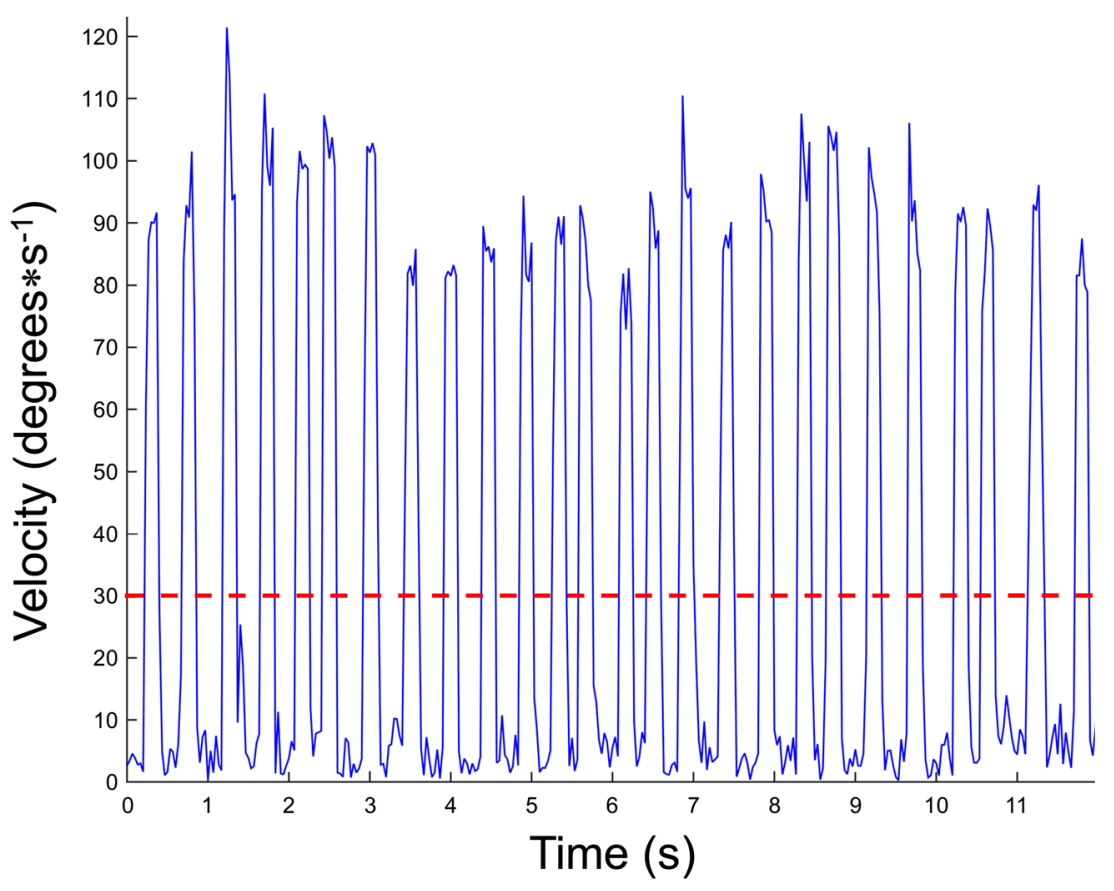

error for both the prosaccade and self-paced tasks, as well as prosaccade latency for the prosaccade tasks, with significantly greater error and latencies, respectively, exhibited by the concussed group compared to the healthy controls. While not significantly different, healthy controls trended toward having performed more self-paced saccades than concussed participants over the same amount of time. Table 2 displays the group differences in the dependent variables for the pursuit tasks. Notably, there were significant differences observed in the phase lag of the $180^{\circ} * \mathrm{~s}^{-1}$ and $360^{\circ} *$ $\mathrm{s}^{-1}$ smooth pursuit task and the random pursuit task conditions, with healthy controls exhibiting significantly decreased lag times (indicated by lower $\mathrm{M}$ values) in tracking

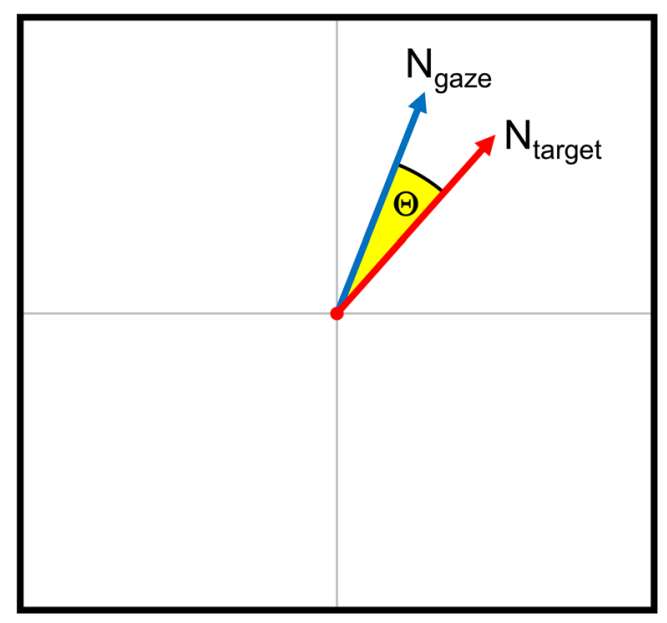

Fig. 6 Calculation of smooth pursuit lag as a function of phase angle. The red arrow represents the phase angle of the target, and the blue arrow represents that phase angle of gaze the target at those velocities. Additionally, healthy controls exhibited significantly less tracking angular velocity for the $180^{\circ} * \mathrm{~s}^{-1}$ condition.

\section{Discussion}

The results obtained in this study provide evidence that the methods proposed are useful for the detection of oculomotor deficits in individuals resulting from closed head injury (i.e., concussion) compared to their healthy peers. Specifically, concussed individuals were significantly less accurate while fixating on a target between saccades and exhibited impaired performance in tracking a target along a predictable path compared to the healthy controls. These results indicate that these measures may have potential to identify adolescents who experience a concussive event. However, more research is needed with larger sample sizes to fully identify the clinical significance of oculomotor performance differences in these groups. In addition, an even more salient finding may be that following such an injury, young athletes incur significant oculomotor deficits that could potentially put these individuals at a disadvantage in settings where quick and accurate interpretation of visual information is imperative for maintaining performance or safety. These deficits may be associated with the high reinjury risk following a primary concussion event (Lynall, Mauntel, Padua, \& Mihalik, 2015). These results also emphasize that systematic analyses of recording and reducing gaze data may be important in developing appropriate and effective oculomotor assessments in oculomotor-driven performance settings, such as playing a sport or operating a vehicle. 
Table 1 Mean, standard deviation, p-values, t-statistics, and $95 \%$ confidence intervals (CIs) of group differences in dependent variables determined for the saccade tasks

\begin{tabular}{|c|c|c|c|c|c|c|c|c|c|c|}
\hline \multirow[b]{2}{*}{ Measure } & \multirow[b]{2}{*}{ Task } & \multicolumn{2}{|c|}{ Concussions } & \multicolumn{2}{|c|}{ Healthy controls } & \multirow[b]{2}{*}{$p$} & \multirow[b]{2}{*}{ t-stat. } & \multirow[b]{2}{*}{ CI Lower } & \multirow[b]{2}{*}{ CI Upper } & \multirow[b]{2}{*}{$d$} \\
\hline & & Mean & SD & Mean & $\mathrm{SD}$ & & & & & \\
\hline \multirow[t]{2}{*}{ Number of saccades } & Prosaccade & - & - & - & - & - & - & - & - & - \\
\hline & Self-paced & 57.38 & 12.88 & 64.18 & 15.91 & .061 & -1.906 & -13.91 & 0.32 & 0.47 \\
\hline \multirow[t]{2}{*}{ Saccade latency (ms) } & Prosaccade & 165.24 & 40.72 & 149.09 & 29.58 & $.003^{*}$ & 3.044 & 5.55 & 26.74 & -0.45 \\
\hline & Self-paced & - & - & - & - & - & - & - & - & \\
\hline \multirow[t]{2}{*}{ Saccade velocity $\left({ }^{0} * \mathrm{~s}^{-1}\right)$} & Prosaccade & 56.52 & 12.36 & 57.69 & 11.60 & .316 & -1.011 & -3.50 & 1.15 & 0.10 \\
\hline & Self-paced & 87.27 & 17.10 & 89.75 & 10.92 & .070 & -1.840 & -5.17 & 0.21 & 0.07 \\
\hline \multirow[t]{2}{*}{ Fixation error $(\mathrm{cm})$} & Prosaccade & 1.11 & 0.35 & 0.86 & 0.26 & $.006^{*}$ & 2.846 & 0.08 & 0.44 & -0.84 \\
\hline & Self-paced & 1.25 & 0.47 & 0.89 & 0.26 & $.002 *$ & 3.228 & 0.14 & 0.58 & -0.98 \\
\hline \multirow[t]{2}{*}{ Initial fixation error (cm) } & Prosaccade & 1.14 & 0.37 & 0.93 & 0.32 & $.022 *$ & 2.334 & 0.03 & 0.39 & -0.61 \\
\hline & Self-paced & 1.40 & 0.60 & 0.99 & 0.35 & $<.001 *$ & 3.371 & 0.19 & 0.62 & -0.84 \\
\hline
\end{tabular}

Note. * Indicates statistical significance.

Saccades have commonly and generally been linked to attention (Hutton, 2008); they allow individuals to quickly and selectively discriminate between objects of interest pertaining to the specific environments or contexts that characterize the visual field (Deubel \& Schneider, 1996) and, accordingly, precede the efficient and accurate fixation of those objects (Kowler, Anderson, Dosher, \& Blaser, 1995). Saccades also serve as a corrective behavior when fixation accuracy is low; specifically, saccades are used to establish visual tracking of a target, with subtle corrections taking place to improve fixation precision. In the case of poor fixation accuracy, an individual would compensate through a greater number of saccades, for example, and this may lead to increased reaction times and less efficient visual search and attention behavior. Smooth pursuits are mechanistically similar, involving a combination of smooth tracking and saccadic movements. Successful pursuit tracking, however, places a greater emphasis on attention, working memory, and predictive processes (Barnes, 2008). Specifically, in tracking a target that is following a predictable and periodic trajectory, successful pursuit is constrained by the individual's ability to obtain and process information relative to the target's trajectory and velocity. In environments where successful performance is dictated by efficient uptake of visual information, as well as the individual's ability to generate an appropriate behavioral response, deficits in oculomotor function due to injury place the individual at risk for diminished performance (Capo-Aponte, Urosevich, Temme, Tarbett, \& Sanghera, 2012), and possible additional injury. Smooth pursuit, prosaccade, and fixation eye measures have been previously shown to be highly reliable within-session (Ettinger et al., 2003); moreover, in the present study, eye-tracking measures were found to be reliable between tests as no significant differences existed among all participants for each task.

That concussed individuals were less accurate in their fixations indicated a diminished saccade performance or capability of attending to important objects, or some combination of

Table 2 Mean, standard deviation, p-values, t-statistics, and $95 \%$ confidence intervals (CIs) of group differences in dependent variables determined for the pursuit tasks

\begin{tabular}{|c|c|c|c|c|c|c|c|c|c|c|c|}
\hline \multirow[b]{2}{*}{ Task } & \multirow[b]{2}{*}{ Angular velocity $\left({ }^{\circ} * \mathrm{~s}^{-1}\right)$} & \multirow[b]{2}{*}{ Measure } & \multicolumn{2}{|c|}{ Concussions } & \multicolumn{2}{|c|}{ Healthy controls } & \multirow[b]{2}{*}{$p$} & \multirow[b]{2}{*}{ t-stat. } & \multirow[b]{2}{*}{ CI lower } & \multirow[b]{2}{*}{ CI upper } & \multirow[b]{2}{*}{$d$} \\
\hline & & & Mean & SD & Mean & SD & & & & & \\
\hline \multirow[t]{6}{*}{ Smooth pursuit } & \multirow[t]{2}{*}{90} & Phase lag $\left(^{\circ}\right)$ & 15.23 & 13.79 & 12.09 & 12.36 & .090 & 1.721 & -.50 & 6.79 & 0.24 \\
\hline & & Gaze velocity $\left({ }^{\circ} * \mathrm{~s}^{-1}\right)$ & 132.42 & 146.36 & 123.08 & 111.36 & .253 & 1.153 & -6.83 & 25.51 & 0.07 \\
\hline & \multirow[t]{2}{*}{180} & Phase lag $\left(^{\circ}\right)$ & 15.84 & 13.67 & 11.57 & 9.24 & $<.001 *$ & 3.939 & 2.10 & 6.43 & 0.37 \\
\hline & & Gaze velocity $\left({ }^{\circ} * \mathrm{~s}^{-1}\right)$ & 231.98 & 203.84 & 208.02 & 125.14 & $.013^{*}$ & 2.554 & 5.22 & 41.70 & 0.15 \\
\hline & \multirow[t]{2}{*}{360} & Phase lag $\left(^{\circ}\right)$ & 22.45 & 16.90 & 16.41 & 11.83 & $<.001 *$ & 3.960 & 2.99 & 9.09 & 0.42 \\
\hline & & Gaze velocity $\left({ }^{*}{ }^{*} \mathrm{~s}^{-1}\right)$ & 410.65 & 323.50 & 408.31 & 239.00 & .775 & 0.288 & 13.89 & 18.56 & 0.01 \\
\hline \multirow[t]{2}{*}{ Random pursuit } & \multirow[t]{2}{*}{ - } & Phase lag $\left(^{\circ}\right)$ & 17.89 & 15.04 & 14.37 & 11.00 & $.013 *$ & 2.548 & 0.76 & 6.27 & 0.27 \\
\hline & & Gaze velocity $\left({ }^{\circ} * \mathrm{~s}^{-1}\right)$ & 263.83 & 250.94 & 261.38 & 214.83 & .822 & 0.226 & 19.33 & 24.24 & 0.01 \\
\hline
\end{tabular}

Note. * Indicates statistical significance. 


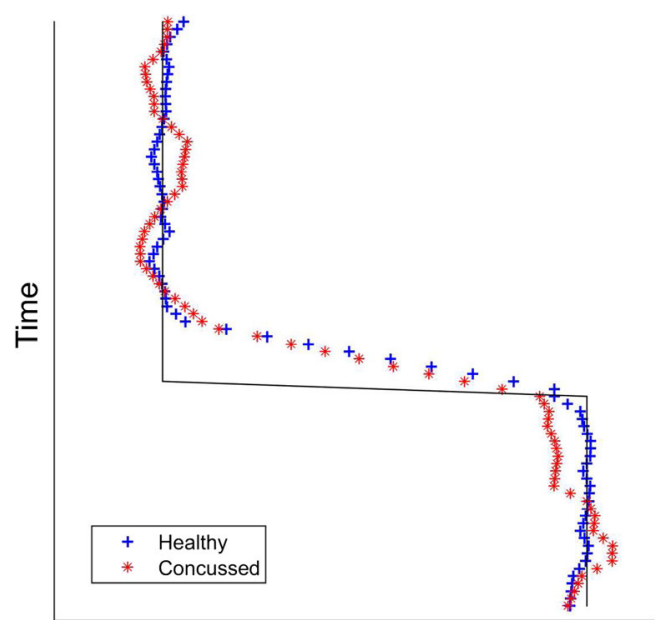

Horizontal Position

Fig. 7 Representative sample showing saccade accuracy for both a healthy (blue) and a concussed (red) participant. The black line represents the horizontal displacement of the center of the target during a saccade event. Both participants initially saccade past the target, represented by the first trajectory peak above the target path, followed by two smaller corrective saccades to center on the target. The amplitude of saccade error is greater in the concussed patient, resulting in diminished fixation accuracy and a temporal shift of fixation on the target

both. Patients who had recently incurred head trauma have been shown previously to exhibit fewer self-paced saccades in a 30-s test (Williams et al., 1997), an association that was trending in the similar task we employed. We did observe a discrepancy in prosaccade latency, which has also been shown in patients with head trauma for a visually guided and memory guided task (Williams et al., 1997). For most individuals, their saccades typically overshoot a target initially, proportionate to distance, and are accompanied by a smaller, corrective saccade to fixate more accurately on the target (Kapoula, 1985). Incidentally, this exhibits a compounding effect on oculomotor function; concussed individuals exhibited a higher initial saccade error, necessitating a larger (and longer) corrective saccade and resulting in a diminished capacity to selectively attend to objects in the visual field. Figure 7 illustrates this scenario for a representative healthy control and concussed participant of the current study. Similarly, in the smooth pursuit tasks, discrepancies in phase lag were observed only at the faster target speeds; given that the number and size of saccades needed to re-align the eyes with the target during a pursuit task increases as a function of target speed (Becker \& Fuchs, 1985), fixation accuracy likely has implications for smooth tracking. Impaired individuals may be less successful than healthy controls at tracking relevant objects moving at sufficient speeds in their visual fields and especially predicting the motion of those objects: a deficit that has been established in individuals with brain injury (Suh et al., 2006b). The random pursuit task, arguably the most cognitively demanding of the pursuit tasks in terms of attention and cognitive processing, also showed a significant discrepancy, which is consistent with previous findings (Heitger et al., 2004), despite the shorter duration of the tasks in the current study compared to those used in previous studies (Heitger et al., 2004).

Of the methods employed in this study, there were some potentially significant limitations that should be addressed. The filtering process may have affected some of our dependent measures - particularly saccade velocity. Although the filtering process we employed was used to assist in more accurate detection of saccades of interest, it is possible that the averaging window we used (five frames) might have overdampened the data. Consequently, the discrepancy in peak saccade velocity between the concussed and healthy controls may have been reduced. It is also important to note that the eye tracker used in the present study only sampled at $60 \mathrm{~Hz}$, as opposed to higher-sampling eye trackers that are typically employed for tasks such as these. In order for this approach to have applicability in clinical settings, it is necessary to provide a low-cost, highly portable system. Therefore, we are encouraged by the current findings given their sensitivity to the differences between groups despite the lower sampling rate, compared to traditional equipment used in similar studies (Heitger et al., 2002, 2004, 2006; Suh et al. 2006a, b).

\section{Conclusion}

This paper presents a reliable way to detect and measure oculomotor deficits in individuals who have recently suffered a closed head injury. For impaired individuals who are slower to respond to, or are less accurate in tracking, visual stimuli especially those that are critical to performance or safe execution of a task - the presence of these deficits may have a negative effect on their well-being and warrant further investigation. A systematic eye-tracking analysis scheme such as the one proposed may prove beneficial for those appointed to ensure the accurate diagnosis and prognosis of these individuals, including physicians and other practitioners.

Acknowledgments This work was funded in part by a state of Ohio Emergency Medical Services grant and also by Q30 Sports Sciences, LLC.

\section{References}

Barnes, G. R. (2008). Cognitive processes involved in smooth pursuit eye movements. Brain and Cognition, 68(3), 309-326. doi:10.1016/j. bandc. 2008.08.020

Becker, W., \& Fuchs, A. F. (1985). Prediction in the oculomotor system: smooth pursuit during transient disappearance of a visual target. Experimental Brain Research, 57(3), 562-575. Retrieved from http://www.ncbi.nlm.nih.gov/pubmed/3979498

Capo-Aponte, J. E., Urosevich, T. G., Temme, L. A., Tarbett, A. K., \& Sanghera, N. K. (2012). Visual dysfunctions and symptoms during the subacute stage of blast-induced mild traumatic brain injury. 
Military Medicine, 177(7), 804-813. Retrieved from http://www. ncbi.nlm.nih.gov/pubmed/22808887

Carroll, L. J., Cassidy, J. D., Peloso, P. M., Borg, J., von Holst, H., Holm, L., ... Injury, W. H. O. C. C. T. F. o. M. T. B. (2004). Prognosis for mild traumatic brain injury: results of the WHO Collaborating Centre Task Force on Mild Traumatic Brain Injury. $J$ Rehabil $\operatorname{Med}(43$ Suppl), 84-105. Retrieved from http://www.ncbi.nlm.nih. gov/pubmed/15083873

Cifu, D. X., Wares, J. R., Hoke, K. W., Wetzel, P. A., Gitchel, G., \& Carne, W. (2015). Differential eye movements in mild traumatic brain injury versus normal controls. The Journal of Head Trauma Rehabilitation, 30(1), 21-28. doi:10.1097/HTR. 0000000000000036

Crawford, T. J., Higham, S., Renvoize, T., Patel, J., Dale, M., Suriya, A., \& Tetley, S. (2005). Inhibitory control of saccadic eye movements and cognitive impairment in Alzheimer's disease. Biological Psychiatry, 57(9), 1052-1060. doi:10.1016/j.biopsych.2005.01.017

Deubel, H., \& Schneider, W. X. (1996). Saccade target selection and object recognition: evidence for a common attentional mechanism. Vision Research, 36(12), 1827-1837. Retrieved from http://www. ncbi.nlm.nih.gov/pubmed/8759451

Ettinger, U., Kumari, V., Crawford, T. J., Davis, R. E., Sharma, T., \& Corr, P. J. (2003). Reliability of smooth pursuit, fixation, and saccadic eye movements. Psychophysiology, 40(4), 620-628. Retrieved from http://www.ncbi.nlm.nih.gov/pubmed/14570169

Grubenhoff, J. A., Kirkwood, M. W., Deakyne, S., \& Wathen, J. (2011). Detailed concussion symptom analysis in a paediatric ED population. Brain Injury, 25(10), 943-949. doi:10.3109/02699052.2011. 597043

Guskiewicz, K. M., McCrea, M., Marshall, S. W., Cantu, R. C., Randolph, C., Barr, W., ... Kelly, J. P. (2003). Cumulative effects associated with recurrent concussion in collegiate football players: the NCAA Concussion Study. JAMA, 290(19), 2549-2555. doi:10. 1001/jama.290.19.2549

Heitger, M. H., Anderson, T. J., \& Jones, R. D. (2002). Saccade sequences as markers for cerebral dysfunction following mild closed head injury. Progress in Brain Research, 140, 433-448. doi:10. 1016/S0079-6123(02)40067-2

Heitger, M. H., Anderson, T. J., Jones, R. D., Dalrymple-Alford, J. C., Frampton, C. M., \& Ardagh, M. W. (2004). Eye movement and visuomotor arm movement deficits following mild closed head injury. Brain, 127(Pt 3), 575-590. doi:10.1093/brain/awh066
Heitger, M. H., Jones, R. D., Dalrymple-Alford, J. C., Frampton, C. M., Ardagh, M. W., \& Anderson, T. J. (2006). Motor deficits and recovery during the first year following mild closed head injury. Brain Injury, 20(8), 807-824. doi:10.1080/02699050600676354

Hutton, S. B. (2008). Cognitive control of saccadic eye movements. Brain and Cognition, 68(3), 327-340. doi:10.1016/j.bandc.2008. 08.021

Kapoula, Z. (1985). Evidence for a range effect in the saccadic system. Vision Research, 25(8), 1155-1157. Retrieved from http://www. ncbi.nlm.nih.gov/pubmed/4071995

Kowler, E., Anderson, E., Dosher, B., \& Blaser, E. (1995). The role of attention in the programming of saccades. Vision Research, 35(13), 1897-1916. Retrieved from http://www.ncbi.nlm.nih.gov/pubmed/ 7660596

Lovell, M. R., Collins, M. W., Iverson, G. L., Field, M., Maroon, J. C., Cantu, R., ... Fu, F. H. (2003). Recovery from mild concussion in high school athletes. J Neurosurg, 98(2), 296-301. doi:10.3171/jns. 2003.98.2.0296

Lynall, R. C., Mauntel, T. C., Padua, D. A., \& Mihalik, J. P. (2015). Acute lower extremity injury rates increase following concussion in college athletes. Medicine and Science in Sports and Exercise. doi:10. 1249/MSS.0000000000000716

Olsen, A. (2012). The Tobii I-VT Fixation Filter: Algorithm description. Tobii Eye Tracking Research Web Site. http://www.tobii.com/en/ eye-tracking-research/global/library/white-papers/the-tobii-i-vtfixation-filter/ Accessed January 17, 2015. Retrieved from http:// www.tobii.com/en/eye-tracking-research/global/library/whitepapers/the-tobii-i-vt-fixation-filter/

Suh, M., Basu, S., Kolster, R., Sarkar, R., McCandliss, B., Ghajar, J., ... Neurobiological Research, C. (2006). Increased oculomotor deficits during target blanking as an indicator of mild traumatic brain injury. Neurosci Lett, 410(3), 203-207. doi:10.1016/j.neulet.2006.10.001

Suh, M., Kolster, R., Sarkar, R., McCandliss, B., Ghajar, J., \& Cognitive, \& Neurobiological Research, C. (2006b). Deficits in predictive smooth pursuit after mild traumatic brain injury. Neuroscience Letters, 401(1-2), 108-113. doi:10.1016/j.neulet.2006.02.074

Williams, I. M., Ponsford, J. L., Gibson, K. L., Mulhall, L. E., Curran, C. A., \& Abel, L. A. (1997). Cerebral control of saccades and neuropsychological test results after head injury. Journal of Clinical Neuroscience, 4(2), 186-196. Retrieved from http://www.ncbi. nlm.nih.gov/pubmed/18638954 